\title{
Rational design of reconfigurable prismatic architected materials
}

\section{Citation}

Overvelde, Johannes T. B., James C. Weaver, Chuck Hoberman, and Katia Bertoldi. 2017. "Rational Design of Reconfigurable Prismatic Architected Materials." Nature 541 (7637) (January 18): 347-352. doi:10.1038/nature20824.

\section{Published Version}

doi:10.1038/nature20824

\section{Permanent link}

http://nrs.harvard.edu/urn-3:HUL.InstRepos:33719365

\section{Terms of Use}

This article was downloaded from Harvard University's DASH repository, and is made available under the terms and conditions applicable to Other Posted Material, as set forth at http:// nrs.harvard.edu/urn-3:HUL.InstRepos:dash.current.terms-of-use\#LAA

\section{Share Your Story}

The Harvard community has made this article openly available.

Please share how this access benefits you. Submit a story.

\section{Accessibility}




\title{
Rational design of reconfigurable prismatic architected materials
}

\author{
Johannes T. B. Overvelde ${ }^{1,2}$, James C. Weaver ${ }^{3}$, Chuck Hoberman ${ }^{3,4,5}$, and Katia \\ Bertoldi ${ }^{1,6}$ \\ ${ }^{1}$ School of Engineering and Applied Sciences, Harvard University, Cambridge, MA \\ 02138, United States \\ ${ }^{2}$ FOM Institute AMOLF, Science Park 104, 1098 XG Amsterdam, The Netherlands \\ ${ }^{3}$ Wyss Institute for Biologically Inspired Engineering, Harvard University, Cambridge \\ , MA 02138, United States \\ ${ }^{4}$ Hoberman Associates, New York, NY 10001, United States \\ ${ }^{5}$ Graduate School of Design, Harvard University, Cambridge, MA 02138, United States \\ ${ }^{6}$ Kavli Institute, Harvard University, Cambridge, MA 02138, United States
}

November 6, 2016

\begin{abstract}
Advances in fabrication technologies are enabling the production of architected materials with unprecedented properties. While most of these materials are characterized by a fixed geometry, an intriguing avenue is to incorporate internal mechanisms capable of reconfiguring their spatial architecture, therefore enabling tunable functionality. Inspired by the structural diversity and foldability of the prismatic geometries that can be constructed using the snapology origami-technique, here we introduce a robust design strategy based on space-filling polyhedra to create 3D reconfigurable materials comprising a periodic assembly of rigid plates and elastic hinges. Guided by numerical analysis and physical prototypes, we systematically explore the mobility of the designed structures and identify a wide range of qualitatively different deformations and internal rearrangements. Given that the underlying principles are scale-independent, our strategy can be applied to design the next generation of reconfigurable structures and materials, ranging from transformable meter-scale architectures to nanoscale tunable photonic systems.
\end{abstract}

\section{Introduction}

In the search for materials with new properties, there have been significant advances in recent years aimed at the construction of architected materials whose behavior is governed by structure, rather than composition. ${ }^{1-3}$ Through careful design of the material's architecture, new material properties have been demonstrated, including negative index of refraction, ${ }^{4,5}$ negative Poisson's ratio, ${ }^{6}$ high stiffness to weight ratio $^{7,8}$ and optical ${ }^{9}$ and mechanical ${ }^{10}$ cloaking. However, most of the proposed architected materials (also known as metamaterials) have a unique structure that cannot be reconfigured after fabrication, making them suitable only for a specific task and limiting their applicability to well-known and controlled environments.

The ancient art of origami provides an ideal platform for the design of reconfigurable systems, since a myriad of shapes can be achieved by actively folding thin sheets along pre-defined creases. While most of the proposed origami-inspired metamaterials are based on 2D folding patterns, such as the miura-ori, ${ }^{11-17}$ the square twist ${ }^{18}$ and the box-pleat tiling, ${ }^{19}$ it has been shown that cellular structures can be designed by stacking these folded layers, ${ }^{13}$ or assembling them in tubes. ${ }^{20-23}$ Furthermore, by 
taking inspiration from snapology 24,25 - a modular origami technique - a highly reconfigurable 3D metamaterial assembled from extruded cubes has been designed. ${ }^{26}$ Although these examples showcase the potential of origami-inspired designs to enable reconfigurable architected materials, they do not fully exploit the range of achievable deformations and only cover a small region of the available design space. As a result, ample opportunities for the design of architected materials with tunable responses remain to be explored.

Here, we introduce a robust strategy to design 3D reconfigurable architected materials and show that a wealth of responses can be achieved in periodic 3D assemblies of rigid plates connected by elastic hinges. To build these structures, we use periodic space-filling tessellations of convex polyhedra as templates, and extrude arbitrary combinations of the polygon faces. In an effort to design architected materials with specific properties, we systematically explore the proposed designs by performing numerical simulations and characterize the mobility (i.e. number of degrees of freedom) of the systems. We find that qualitatively different responses can be achieved, including shear, uniform expansion along one or two principal directions, and internal reconfigurations that do not alter the macroscopic shape of the materials. Therefore, this research paves the way for a new class of structures that can tune their shape and function to adapt and even influence their surroundings, bringing origami-inspired metamaterials closer to application.

\section{Design Strategy}

To design 3D reconfigurable architected materials, we start by selecting a space-filling and periodic assembly of convex polyhedra (Fig. 1). We then perform two operations on the tessellation: $(i)$ we separate adjacent polyhedra while ensuring that the normals of the overlapping faces remain aligned. This can be achieved by imposing that for each overlapping face pair

$$
d \mathbf{p}_{j, \mathrm{~b}}-d \mathbf{p}_{j, \mathrm{a}}=2 L_{j} \mathbf{n}_{j},
$$

where $d \mathbf{p}_{j}$ denotes the displacements applied to the polyhedra to separate them, and the subscripts $\mathrm{a}$ and $\mathrm{b}$ indicate to which polyhedron the two overlapping faces belong. Moreover, $L_{j}$ is the distance between the faces in the separated state, and $\mathbf{n}_{j}$ is the unit normal to the face pointing outward of the polyhedron indicated by the subscript a.

(ii) we extrude the edges of the polyhedra in the direction normal to their faces to form a connected thin-walled structure (Fig. 1), which we refer to as a prismatic architected material (Supplementary Movie 1).

Importantly, for the periodic space-filling tessellations considered here, it is sufficient to focus on a unit cell that consists of only a few polyhedra and covers the entire assembly when translated by the three lattice vectors $\mathbf{l}_{i}^{0}(i=1,2,3)$. While Eq. (1) can be directly imposed to all internal face pairs in the unit cell, for overlapping faces that are periodically located (i.e. lie on the external boundary of the unit cell) the constraint needs to be updated as

$$
d \mathbf{p}_{j, \mathrm{~b}}-d \mathbf{p}_{j, \mathrm{a}}+\mathbf{R}_{j}-\mathbf{R}_{j}^{0}=2 L_{j} \mathbf{n}_{j},
$$

where $\mathbf{R}_{j}=\sum_{i=1}^{3} \alpha_{j, i} \mathbf{l}_{i}$ and $\mathbf{R}_{j}^{0}=\sum_{i=1}^{3} \alpha_{j, i} \mathbf{l}_{i}^{0}$ denote the distance between the two periodically located faces in the expanded and initial configuration, respectively, $\mathbf{l}_{i}$ being the lattice vectors of the expanded unit cell and $\alpha_{j, i} \in\{-1,0,1\}$. As shown by Eqs. (1) and (2), for a unit cell with $F$ face pairs the expanded configuration is fully described by $F$ extrusion lengths $L_{j}(j=1, \ldots, F)$ (Fig. 1$)$. However, for most unit cells the extrusion lengths cannot all be specified independently due to the constraints introduced by by Eqs. (1) and (2). As a result, each unit cell is characterized by $F_{\text {indep }} \leq F$ independent extrusion lengths as illustrated in Supplementary Fig. 6. For the sake of convenience we chose the $F_{\text {indep }}$ independent extrusion lengths to be as close as possible to an average extrusion length, $\bar{L}$, by solving

$$
\min _{L_{1} \ldots L_{F_{\text {indep }}}} \sum_{j=1}^{F}\left(L_{j}-\bar{L}\right)^{2},
$$


while ensuring that the constraints imposed by Eqs. (1) and (2) are not violated.

Finally, we note that all periodic and space-filling assemblies of convex polyhedra tested in this study were successfully extruded following the proposed design strategy (i.e. we always found $F_{\text {indep }} \geq 1$ ). As an example, in Fig. 1 we show three prismatic architected materials based on unit cells containing (a) two triangular and one hexagonal prism, (b) an octahedron and cuboctahedron, and (c) four triangular prisms.

\section{Characterizing Reconfigurability}

Although the aforementioned design strategy represents a robust and efficient approach to construct prismatic architected materials, it does not provide any indication on their reconfigurability. To determine if, and to what extent, the meso-structure of the designed architected materials can be reshaped, we started by fabricating centimeter-scale prototypes from cardboard and double-sided tape (Figs. 2a-c), using a stepwise layering and laser-cutting technique (see Supplementary Information: Methods). ${ }^{26,27}$

Focusing on the three architected materials shown in Fig. 1, we find that the structure based on triangular prisms and the one based on a combination of triangular and hexagonal prisms can be reconfigured by bending the edges and without deforming the faces, and are respectively characterized by one and two deformation modes (Figs. 2d-e and Supplementary Movie 2). In contrast, the material based on a combination of octahedra and cuboctahedra is completely rigid (Fig. 2b and Supplementary Movie 2). Furthermore, our experiments reveal that these architected materials have fewer degrees of freedom than their constituent individual extruded polyhedra (Supplementary Fig. 7), indicating that the additional constraints introduced by the connections between the polyhedra effectively reduce their reconfigurability.

\section{Numerical Algorithm}

While the examples of Figs. 2a-e illustrate the potential of our strategy to design reconfigurable architected materials, they also show that the design of systems with specific behavior is not straightforward. To improve our understanding of the reconfigurability of the proposed architected materials, we implemented a numerical algorithm that predicts their mobility and corresponding deformation modes. In our numerical analysis, each extruded unit cell is modeled as a set of rigid faces connected by linear torsional springs, with periodic boundary conditions applied to the vertices located on the boundaries. To characterize the mobility of the structure we solved the following eigenproblem $\tilde{\mathbf{M}}^{-1} \tilde{\mathbf{K}} \mathbf{a}_{\mathrm{m}}=\omega^{2} \mathbf{a}_{\mathrm{m}}$, in which $\tilde{\mathbf{M}}$ and $\tilde{\mathbf{K}}$ are respectively the mass and stiffness matrices, which account for both the rigidity of the faces and the periodic boundary conditions through master-slave elimination. Moreover, $\omega$ is an eigenfrequency of the system and $\mathbf{a}_{\mathrm{m}}$ is the amplitude of the corresponding mode (see Supplementary Information: Mode analysis for $3 D$ prismatic architected materials with rigid faces).

Figs. 2f-g show the simulated eigenmodes for the two reconfigurable architected materials considered in Figs. 1a and c. Although the simulations only predict the deformation for small rotations, the modes are strikingly similar to the deformation observed in the experiments (Figs. 2d-e). Solving the aforementioned eigenproblem therefore provides a convenient approach to determine the mobility of the structures and gives insight into their deformation without the need for specific boundary conditions.

\section{Designs Based on Uniform Tessellations}

To further explore the potential of prismatic architected materials, and to establish relations between their reconfigurability and the initial space-filling polyhedral assembly, we next focus on extruded materials based on the 28 uniform tessellations of the 3D space, which comprise regular polyhedra, semiregular polyhedra and semiregular prisms. ${ }^{28-30}$ Due to their relative simplicity, these uniform templates provide a convenient starting point to explore the design space. 
Using the numerical algorithm, we first determined the number of degrees of freedom, $n_{\text {dof }}$, of the resulting 28 architected materials (Supplementary Fig. 9). We find that the mobility of the unit cells is affected by two parameters: the average connectivity of the unit cell, $\bar{z}=\frac{1}{P} \sum_{p=1}^{P} z_{p}$, and the average number of modes of the individual polyhedra, $\bar{n}=\frac{1}{P} \sum_{p=1}^{P} n_{p}$, where $P$ is the number of polyhedra in the unit cell and $z_{p}$ and $n_{p}$ are the number of extruded faces and modes of the $p$-th polyhedron, respectively (Supplementary Fig. 8). The results for the 28 architected materials reported in Fig. 3 show three key features. First, higher values for $\bar{z}$ lead to rigid materials (i.e. $n_{\text {dof }}=0$ for $\bar{z}>8$ ). Second, if all the constituent extruded polyhedra are rigid (i.e. $\bar{n}=0$ ), the resulting architected material is rigid as well, and third, only 13 of the 28 designs are reconfigurable (i.e. $n_{\text {dof }}>0$ ).

Interestingly, we find that all of the 13 reconfigurable structures are based on unit cells comprising only prisms, such that they recover the relation previously demonstrated for extruded individual prisms, $\bar{n}=\bar{z}-3 .{ }^{31}$ Moreover, our results indicate that most of the reconfigurable structures are characterized by fewer degrees of freedom than the constituent individual polyhedra (i.e. $n_{\text {dof }}<\bar{n}$ ), with the exception of the architected materials based on the cube (\#22) and the triangular prism (\#11) for which $n_{\text {dof }}=\bar{n}$.

Having determined the number of modes for the 28 architected materials, we next characterize the macroscopic deformation associated to each of them. More specifically, we determine the macroscopic volumetric strain $\delta=\sum_{j=1}^{3} \epsilon_{j}$ for each mode, where $\epsilon_{j}$ are the macroscopic principal strains (see Supplementary Information: Mode analysis for $3 D$ prismatic architected materials with rigid faces). Interestingly, we find that for the 13 reconfigurable architectures all modes are characterized by $\delta=0$, which indicates pure macroscopic shearing deformation, as also confirmed by visual inspection of the modes (Supplementary Fig. 9).

To characterize the reconfigurability of prismatic architected materials, so far we assumed the faces to be completely rigid and the hinges to act as linear torsional springs. However, fabrication will always result in deformable faces, leaving the question whether prismatic architected materials can be reconfigured when their faces are deformable. To explore this direction, we updated our numerical algorithm by introducing a set of springs to account for the deformability of the faces ${ }^{12,13,21}$ (see Supplementary Information: Stiffness of $3 D$ prismatic architected materials with deformable faces). We then deformed the extruded unit cells uniaxially and investigated their macroscopic stiffness for different loading directions (identified by the two angles $\gamma$ and $\theta$ as shown in Fig. 4).

In Fig. 4 we report the normalized stiffness $K / E$ as a function of $\gamma$ and $\theta$ for four prismatic architected materials characterized by $t / \bar{L}=0.01, E$ being the Young's modulus of the material and $t$ the thickness of the faces. We find that the response of the architected material based on template $\# 28$, which was previously qualified as rigid (i.e. $n_{\text {dof }}=0$ ), is fairly isotropic as its stiffness does not vary much as a function of the loading direction (i.e. $3.1 \cdot 10^{-3} \leq K / E \leq 4.0 \cdot 10^{-3}$ ). In contrast, the stiffness of architected materials for which $n_{\text {dof }}>0$ drops significantly for specific directions (i.e. $K_{\min } / K_{\max }=\mathcal{O}\left(10^{-3}\right)$ ). Interestingly, these are the loading directions for which the reconfiguring modes get activated, as indicated by the snapshots shown in Fig. 4. Therefore, these results indicate that the deformation modes we found in the limit of rigid faces still persist even when the faces are deformable. Note that we used the same stiffness for bending of the faces and bending of the hinges, and from the results we can therefore conclude that the architecture of these systems make bending of the faces energetically costly (as it is typically accompanied by stretching and shearing of the faces). Finally, materials characterized by higher $n_{\text {dof }}$ are characterized by more "soft directions". As such, materials with $n_{\text {dof }}=1$ seem most promising for the design of reconfigurable architected materials, since they can be reconfigured along a specific direction, while still being able to carry loads in all other directions (Fig. 4 and Supplementary Fig. 10).

\section{Enhancing the Reconfigurability}

Although we have shown that by extruding the edges of expanded assemblies of polyhedra we can construct reconfigurable architected materials, our results indicate that the mobility of the resulting 
structures is significantly reduced by their connectivity. Furthermore, the modes of all reconfigurable designs show a qualitatively similar shearing deformation. To overcome these limitations, we next introduce an additional step in the design strategy and reduce the connectivity of the materials by extruding some of faces of the unit cell, while making the remaining faces rigid.

As an example, in Fig. 5 we consider the architected material based on a tessellation of truncated octahedra (\#28). When all faces are extruded, $\bar{z}=14$, leaving the structure rigid (i.e. $n_{\text {dof }}=0$ ). However, by making 8 of the 14 faces rigid instead of extruding them (Fig. 5a and Supplementary Movie 3) we can reduce the connectivity to $\bar{z}=6$ and the resulting architected material is no longer rigid, as $n_{\text {dof }}=1$. As shown in Fig. 5b and Supplementary Movie 3, this response was also confirmed experimentally. Finally, we note that by varying the face pairs in the unit cell that are made rigid instead of extruded, a total of $2^{F}=128$ different architected materials can be designed using the truncated octahedra as a template. However, only 82 combinations are possible (as all the other cases will result in structures with disconnected parts) and out of those designs only four are reconfigurable. Due to symmetries in the truncated octahedron, these four configurations are identical to the one shown in Fig. 5.

Next, to determine the range of deformations that can be achieved in the proposed structures, we apply the same brute force strategy to the other 27 uniform space-filling tessellations depicted in Fig. 3. For this study we considered a maximum of $2^{16}$ designs per tessellation, randomly selected from the $2^{F}$ possibilities, so that for 11 of the tessellations (\#4-5, \#9-10, \#16-17, \#20-21, \#23, \#25, and \#27) the results are not complete, but rather indicate a trend. Note that we expanded the number of possible designs by removing the polyhedra for which all faces have been made rigid from the extruded unit cell, as those would have resulted in rigid parts completely disconnected from the architected materials.

Of the approximately $0.6 \cdot 10^{6}$ connected designs investigated here (Supplementary Table 1), 90\% are rigid (i.e. $n_{\text {dof }}=0$ ) while the other $10 \%$ are reconfigurable (i.e. $n_{\text {dof }}>0$ ). Supplementary Figs. $11 \mathrm{a}-\mathrm{b}$ show that to achieve reconfigurability we still need $\bar{z} \leq 8$, with the exception of 6 designs based on \#5 for which $\bar{z}=9$ (see Supplementary Fig. 12). Moreover, fully extruded architected materials characterized by $\bar{n}=0$ always remain rigid, independent of the reduced number of connections. Finally, and perhaps more importantly, we also find that using this design approach the mobility of the architected materials can be significantly enhanced, as $0 \leq n_{\text {dof }} \leq 16$ and for many of the structures $n_{\text {dof }}>\bar{n}$ (Supplementary Table 1).

Inspection of the modes also reveals that a variety of qualitatively different types of deformation can be achieved. To better characterize them, in Fig. 6 and Supplementary Fig. 11c-f we report the magnitude of the principal strains, $\|\epsilon\|=\sqrt{\sum_{i=1}^{3} \epsilon_{i}^{2}}$, versus the volumetric strain, $\delta$, for each deformation mode observed in the reconfigurable architected materials investigated here. Interestingly, we find that for many modes $\|\epsilon\|=\delta=0$. These modes do not alter the global shape of the structure, but only result in internal rearrangements. Design \#a shown in Fig. 6 is an example of a structure undergoing such a local deformation. Here, most of the structure is rigid except for 1D tubes that can deform independently. Differently, \#b is an example in which the whole internal structure is deforming, while maintaining the same macroscopic shape (Supplementary Movie 4).

Besides these local modes, Fig. 6 also indicates that there are designs capable of achieving types of macroscopic deformation different than pure shear (for which $\delta=0$ and $\|\epsilon\|>0$ ). For example, we find that some of the structures are characterized by an effective vanishing strain in two directions $(\# \mathrm{c})$. The deformation of such architected materials is characterized by $\epsilon_{1} \neq 0$ and $\epsilon_{2}=\epsilon_{3} \approx 0$, resulting in $\delta=\|\epsilon\|$. Moreover, the results also reveal that there are a variety of structures capable of uniform bi-axial expansion (or contraction), for which $\epsilon_{2}=\epsilon_{3} \neq 0$ and $\epsilon_{1}=0$ and $\|\epsilon\|=\delta / \sqrt{2}$. Such deformation mode is exemplified by design \#d shown in Fig. 6 (Supplementary Movie 4). Finally, we note that $\delta=\sqrt{3}\|\epsilon\|$ corresponds to uniform expansion (or contraction) characterized by $\epsilon_{1}=\epsilon_{2}=\epsilon_{3}$, and defines a boundary for possible combinations of $\delta$ and $\|\epsilon\|$. In fact, none of the designs considered here exhibits this type of deformation. 


\section{Discussion and Conclusion}

In this work we introduced a convenient and robust strategy to design reconfigurable architected materials, and explored the design space by considering structures based on the 28 uniform spacefilling tessellations of polyhedra. While our study uncovered architected materials with a wide range of qualitatively different responses and degrees of freedom, many more designs are possible by using different assemblies of convex polyhedra as templates (including assemblies based on Johnson Solids and irregular polyhedra, and assemblies that do not fill space), by considering different extrusion lengths, or by removing faces (instead of making them rigid prior to the extrusion step). Given these additional possibilities in the design of reconfigurable architected materials, we made our numerical algorithm implemented in Matlab available for download as Supplementary Information, to be used and expanded upon by the community. Finally, we believe that, building on the results presented in this work, prismatic architected materials with specific properties may be efficiently identified by combining our numerical algorithm with stochastic optimization algorithms such as Genetic Algorithms. Such optimization algorithms could prove essential in the design of reconfigurable architected materials capable of handling changing environments or multiple task (that will likely lead to pareto optimal solutions).

To realize prismatic architected materials, in this study we used cardboard for the rigid faces and double-sided tape for the hinges. While this fabrication process enables the realization of centimeterscale prototypes (for our models we used $\bar{L}=35 \mathrm{~mm}$ ) that closely match the conceptual origamiinspired mechanisms, real world applications depend on the ability to efficiently manufacture assemblies comprising a large number of unit cells at different length scales using different fabrication techniques. Taking advantage of recent developments in multi-material additive manufacturing, we also built the proposed architected materials using a stiff material (with Young's modulus $E \approx 1 \mathrm{GPa}$ ) for the faces and a soft material $(E \approx 1 \mathrm{MPa}$ ) for the hinges (see Supplementary Information: Methods). Supplementary Movie 5 shows 3D printed models for two designs based on assemblies of truncated octahedra (for both models we used $\bar{L}=6 \mathrm{~mm}$ ). Although additional local deformation arises from the finite size of the flexible hinges, the 3D printed structures exhibit the same deformation modes predicted by our numerical analysis and observed in the cardboard prototypes. As such, recent advances in fabrication, including projection micro-stereolithography, ${ }^{7}$ two-photon lithography ${ }^{8,32,33}$ and 'pop-up' strategies, ${ }^{34-40}$ open exciting opportunities for miniaturization of the proposed architectures. All together, our strategy enables the design of a new class of reconfigurable systems across a wide range of length scales that is waiting to be explored.

Data Availability The Matlab model used to determine the mobility and deformation modes of the prismatic architected materials is provided as Supplementary Information. Other models and datasets generated during and/or analysed during the current study are available from the corresponding author on request.

Acknowledgment This work was supported by the Materials Research Science and Engineering Center under NSF Award No. DMR-1420570. K.B. also acknowledges support from the National Science Foundation (CMMI-1149456-CAREER). We thank Martin Mixe and Simon Shuham for assistance in fabrication of the cardboard prototypes, and Robert Wood for generous use of his lab.

Author Contributions J.T.B.O., C.H. and K.B. proposed and designed the research; J.T.B.O. performed the numerical calculations; J.T.B.O., C.H. and J.C.W. designed and fabricated the models; J.T.B.O. performed the experiments; J.T.B.O. and K.B. wrote the paper.

Permissions Reprints and permissions information is available at www.nature.com/reprints.

Competing interests The authors declare no competing financial interets.

Correspondence Correspondence and requests for materials should be addressed to bertoldi@seas.harvard.edu. 


\section{References}

[1] Reis, P. M., Jaeger, H. M. \& van Hecke, M. Designer matter: A perspective. Extreme Mech. Lett. 5, 25-29 (2015).

[2] Sarrao, J. \& Crabtree, G. Progress in mesoscale science. MRS Bull. 40, 919-922 (2015).

[3] Christensen, J., Kadic, M., Kraft, O. \& Wegener, M. Vibrant times for mechanical metamaterials. MRS Commun. 5, 453-462 (2015).

[4] Pendry, J. B. Negative refraction makes a perfect lens. Phys. Rev. Lett. 85, 3966-3969 (2000).

[5] Smith, D. R., Pendry, J. B. \& Wiltshire, M. C. K. Metamaterials and negative refractive index. Science 305, 788-792 (2004).

[6] Lakes, R. Foam structures with a negative poisson's ratio. Science 235, 1038-1040 (1987).

[7] Zheng, X. et al. Ultralight, ultrastiff mechanical metamaterials. Science 344, 1373-1377 (2014).

[8] Meza, L. R., Das, S. \& Greer, J. R. Strong, lightweight, and recoverable three-dimensional ceramic nanolattices. Science 345, 1322-1326 (2014).

[9] Ergin, T., Stenger, N., Brenner, P., Pendry, J. B. \& Wegener, M. Three-dimensional invisibility cloak at optical wavelengths. Science 328, 337-339 (2010).

[10] Bückmann, T., Kadic, M., Schittny, R. \& Wegener, M. Mechanical cloak design by direct lattice transformation. Proc. Natl. Acad. Sci. U.S.A. 112, 4930-4934 (2015).

[11] Miura, K. Method of packaging and deployment of large membranes in space report No. 618. (Institute of Space and Astronautical Science, 1985).

[12] Wei, Z. Y., Guo, Z. V., Dudte, L., Liang, H. Y. \& Mahadevan, L. Geometric mechanics of periodic pleated origami. Phys. Rev. Lett. 110, 215501 (2013).

[13] Schenk, M. \& Guest, S. D. Geometry of miura-folded metamaterials. Proc. Natl. Acad. Sci. U.S.A. 110, 3276-3281 (2013).

[14] Silverberg, J. L. et al. Using origami design principles to fold reprogrammable mechanical metamaterials. Science 345, 647-650 (2014).

[15] Lv, C., Krishnaraju, D., Konjevod, G., Yu, H. \& Jiang, H. Origami based mechanical metamaterials. Sci. Rep. 4, 5979 (2014).

[16] Waitukaitis, S., Menaut, R., Chen, B. G. \& van Hecke, M. Origami multistability: From single vertices to metasheets. Phys. Rev. Lett. 114, 055503 (2015).

[17] Dudte, L. H., Vouga, E., Tachi, T. \& Mahadevan, L. Programming curvature using origami tessellations. Nat. Mater. 15, 583-588 (2016).

[18] Silverberg, J. L. et al. Origami structures with a critical transition to bistability arising from hidden degrees of freedom. Nat. Mater. 14, 389-393 (2015).

[19] Hawkes, E. et al. Programmable matter by folding. Proc. Natl. Acad. Sci. U.S.A. 107, 1244112445 (2010).

[20] Tachi, T. \& Miura, K. Rigid-foldable cylinders and cells. J. Int. Assoc. Shell Spat. Struct. 53, $217-226$ (2012).

[21] Filipov, E. T., Tachi, T. \& Paulino, G. H. Origami tubes assembled into stiff, yet reconfigurable structures and metamaterials. Proc. Natl. Acad. Sci. U.S.A. 112, 12321-12326 (2015). 
[22] Cheung, K. C., Tachi, T., Calisch, S. \& Miura, K. Origami interleaved tube cellular materials. Smart Mater. Struct. 23, 094012 (2014).

[23] Yasuda, H. \& Yang, J. Re-entrant origami-based metamaterials with negative poisson's ratio and bistability. Phys. Rev. Lett. 114, 185502 (2015).

[24] Goldman, F. Using the snapology technique to teach convex polyhedra. in Origami 5. eds. WangIverson, P., Lang, R. \& Yim, M. (CRC Press, 2011).

[25] Strobl, H. Special snapology. Available at: http://www.knotology.eu/ppp-jena2010e/start.html (2010).

[26] Overvelde, J. T. B. et al. A three-dimensional actuated origami-inspired transformable metamaterial with multiple degrees of freedom. Nat. Commun. 7, 10929 (2016).

[27] Felton, S., Tolley, M., Demaine, E., Rus, D. \& Wood, R. A method for building self-folding machines. Science 345, 644-646 (2014).

[28] Andreini, A. Sulle reti di poliedri regolari e semiregolari e sulle corrispondenti reti correlative. Mem. Società Italiana della Scienze 14, 75-129 (1907).

[29] Pearce, P. \& Pearce, S. Polyhedra Primer (Nostrand Reinhold company, 1978).

[30] Grun̈baum, B. Uniform tilings of 3-space. Geombinatorics 4, 49-56 (1994).

[31] Laliberté, T. \& Gosselin, C. Construction, mobility analysis and synthesis of polyhedra with articulated faces. J. Mechanisms Robotics 6, 011007 (2013).

[32] Bauer, J., Hengsbach, S., Tesari, I., Schwaiger, R. \& Kraft, O. High-strength cellular ceramic composites with 3d microarchitecture. Proc. Natl. Acad. Sci. U.S.A. 111, 2453-2458 (2014).

[33] Meza, L. R. et al. Resilient 3d hierarchical architected metamaterials. Proc. Natl. Acad. Sci. U.S.A. 112, 11502-11507 (2015).

[34] Leong, T. G. et al. Tetherless thermobiochemically actuated microgrippers. Proc. Natl. Acad. Sci. U.S.A. 106, 703-708 (2009).

[35] Liu, Y., Boyles, J. K., Genzer, J. \& Dickey, M. D. Self-folding of polymer sheets using local light absorption. Soft Matter 8, 1764-1769 (2012).

[36] Laflin, K. E., Morris, C. J., Muqeem, T. \& Gracias, D. H. Laser triggered sequential folding of microstructures. Appl. Phys. Lett. 101, (2012).

[37] Shenoy, V. B. \& Gracias, D. H. Self-folding thin-film materials: From nanopolyhedra to graphene origami. MRS Bull. 37, 847-854 (2012).

[38] Cho, J. H. et al. Nanoscale origami for 3d optics. Small 7, 1943-1948 (2011).

[39] $\mathrm{Xu}$, S. et al. Assembly of micro/nanomaterials into complex, three-dimensional architectures by compressive buckling. Science 347, 154-159 (2015).

[40] Kim, J., Hanna, J. A., Byun, M., Santangelo, C. D. \& Hayward, R. C. Designing responsive buckled surfaces by halftone gel lithography. Science 335, 1201-1205 (2012). 


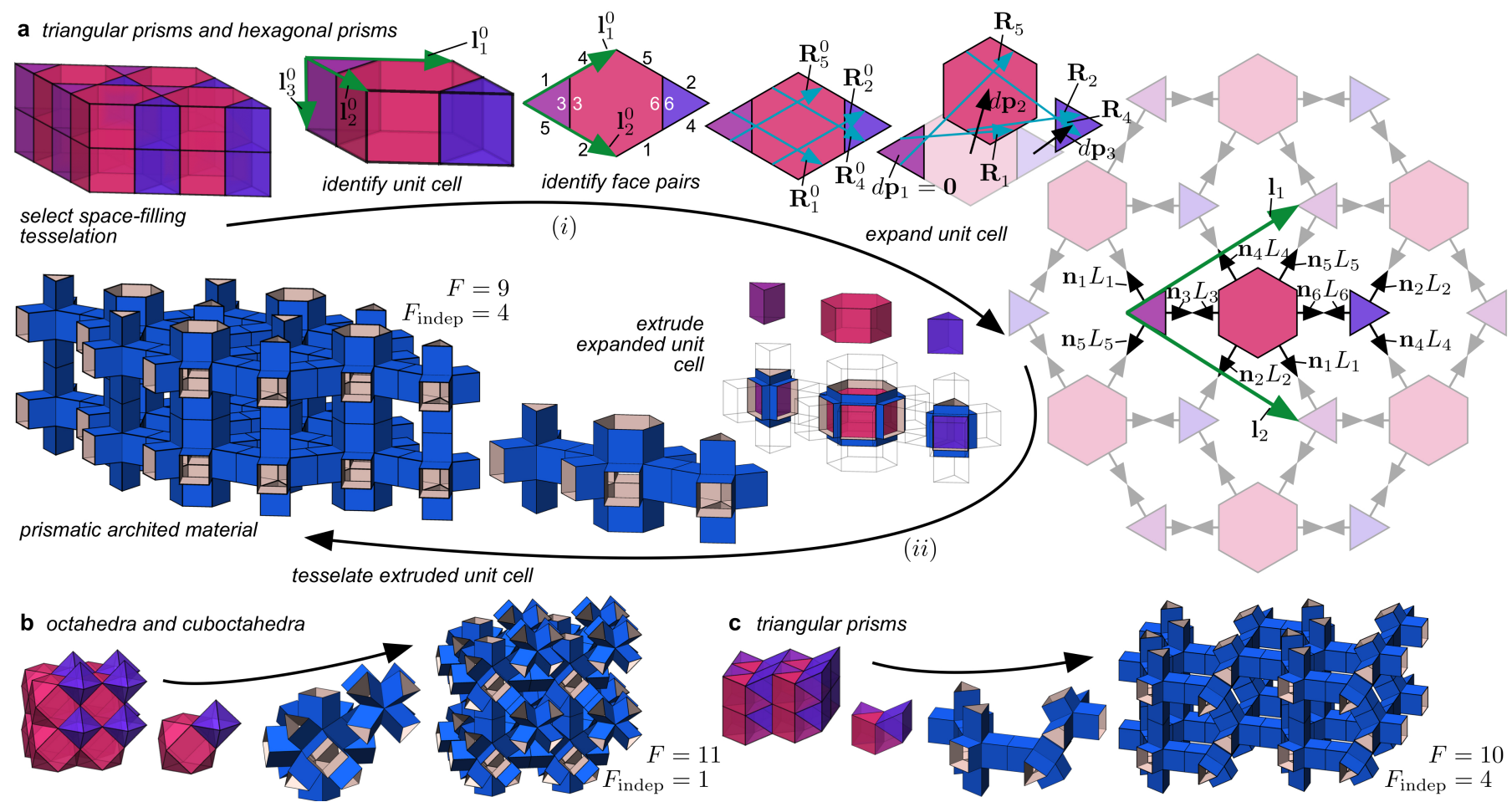

Figure 1: Design strategy to construct 3D prismatic architected materials. Space-filling and periodic assemblies of convex polyhedra are used as a template to construct prismatic architected materials (Supplementary Movie 1). After selecting a space-filling tessellation, we focus on a unit cell spanned by the three lattice vectors $\mathbf{l}_{i}^{0}(i=1,2,3)$ and identify all pairs of overlapping faces. We then separate the polyhedra while ensuring that the normals of all face pairs remain aligned. Finally, we extrude the edges of the polyhedra in the direction normal to their faces to construct the extruded unit cell. Note that the architected material can be constructed by tessellating the extruded unit cell along the three new lattice vectors $\mathbf{l}_{i}$. Using this approach, we designed three architected materials that are based on space-filling tessellations comprising (a) triangular prisms and hexagonal prisms, (b) octahedra and cuboctahedra, and (c) triangular prisms. 


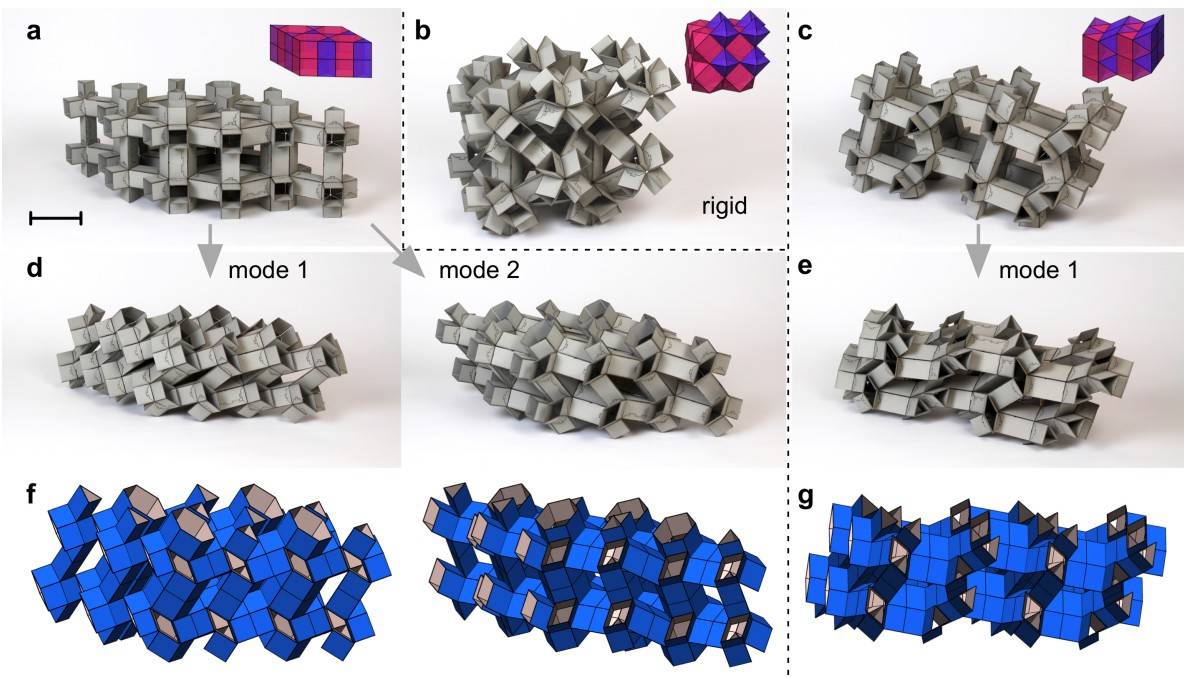

Figure 2: Deformation modes of 3D prismatic architected materials. (a-c) Prototypes of the 3D prismatic architected materials shown in Fig. 1 were constructed using cardboard (rigid faces) and double-sided tape (flexible hinges). (d) The structure based on a combination of triangular and hexagonal prisms can be reconfigured in two different ways (i.e. has two degrees of freedom). (e) The structure based on triangular prisms has a single deformation mode. Note that the architected material based on the octahedra and cuboctahedra cannot be reconfigured. (f-g) Simulated modes of the reconfigurable architected materials. The obtained deformation modes were linearly scaled to match the experiments (scale bar $10 \mathrm{~cm}$ ).

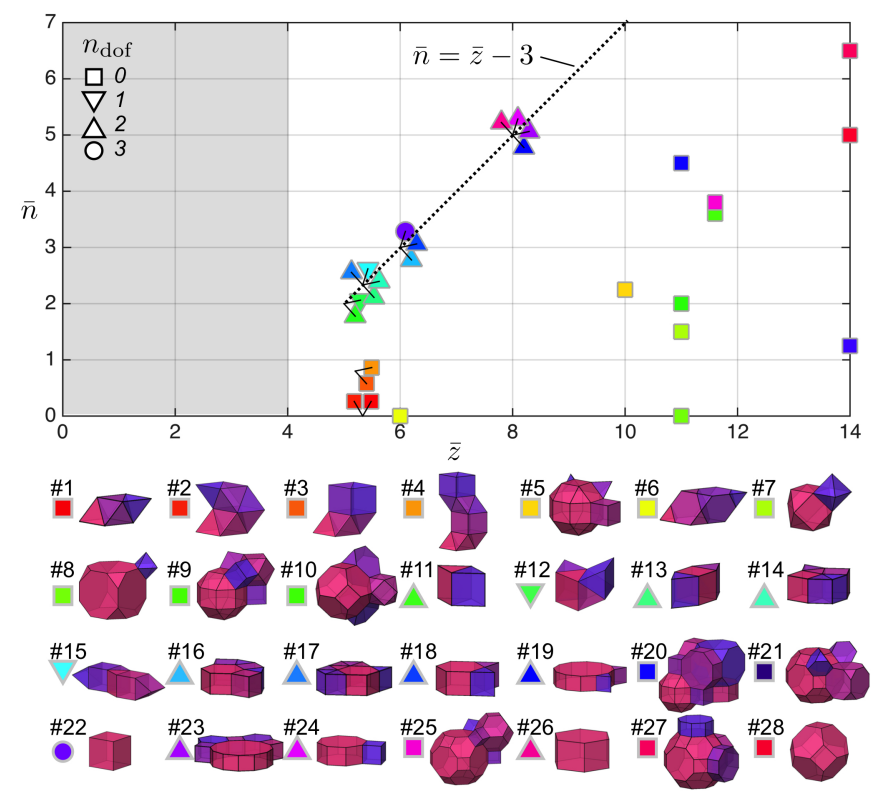

Figure 3: Number of degrees of freedom for architected materials based on the 28 uniform tessellations of the $3 \mathrm{D}$ space. The mobility of the structures is affected by the average connectivity, $\bar{z}$, and the average mobility, $\bar{n}$. The prismatic architected materials and their deformation modes are shown in Supplementary Fig. 9. 

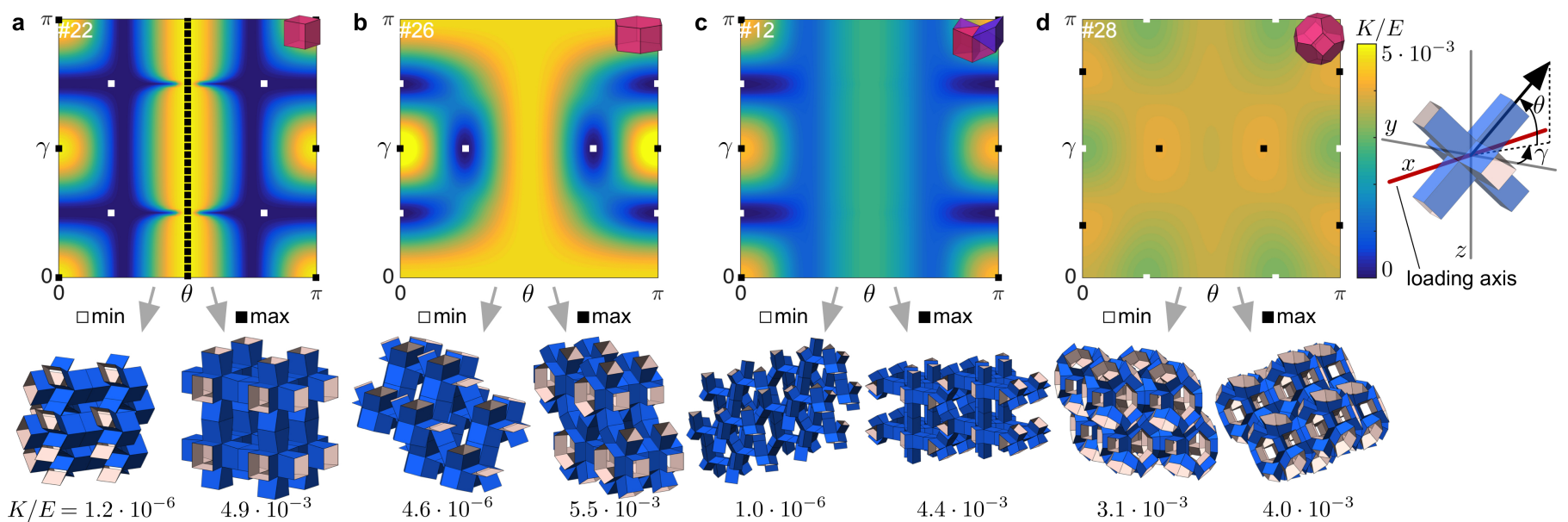

Figure 4: Normalized stiffness $K / E$ of prismatic architected materials. The figure shows the results for architected materials based on template (a) \#22, (b) \#26, (c) \#12, and (d) \#28. To determine the stiffness in all loading directions, the architected materials are rotated by angles $\gamma$ and $\theta$ prior to loading. In each contour plot we indicate the minimum and maximum stiffness with white and black squares, respectively. Moreover, we also show the deformed architected materials for the mimimum and maximum stiffness direction. Note that the deformation is magnified to facilitate visualization.

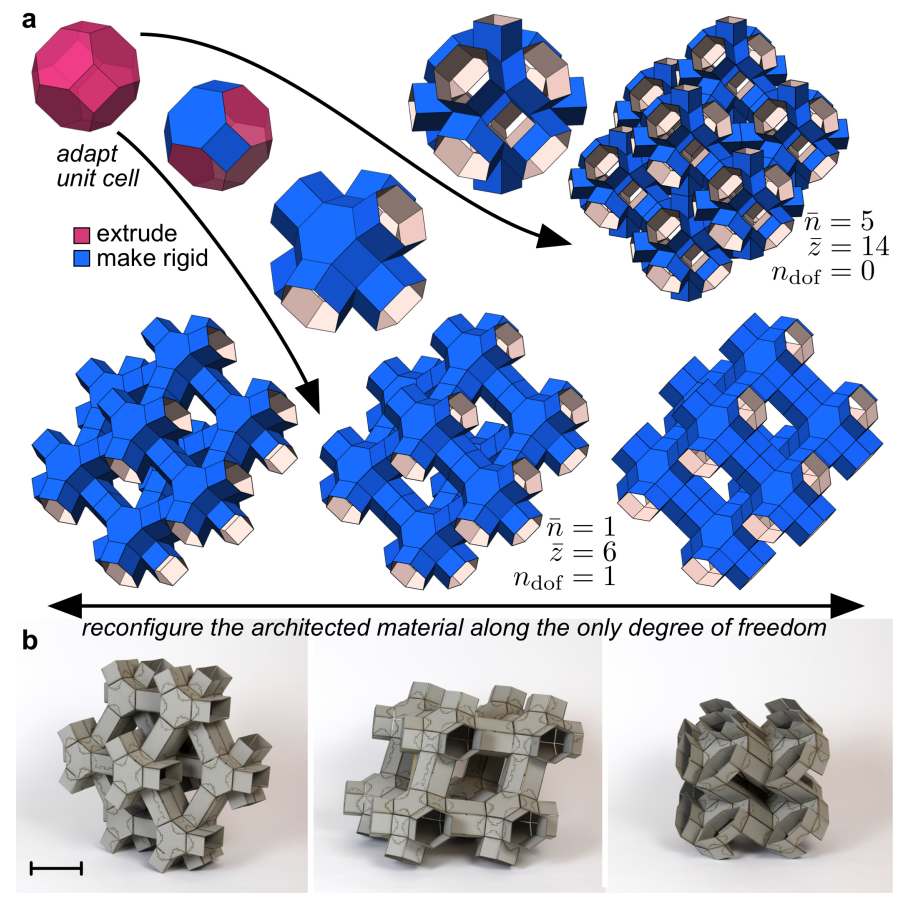

Figure 5: Enhancing the reconfigurability of 3D prismatic architected materials. (a) To enhance the reconfigurability of the architected material based on the space-filling assembly of truncated octahedra (\#11 in Fig. 3), we extrude only 6 of its faces and make the remaining 8 faces rigid. Using this approach, the average connectivity is reduced from $\bar{z}=14$ to $\bar{z}=6$ and the resulting structure is no longer rigid, as $n_{\text {dof }}=1$. (b) Experimental validation of the numerical predictions (scale bar $10 \mathrm{~cm}$ ). 

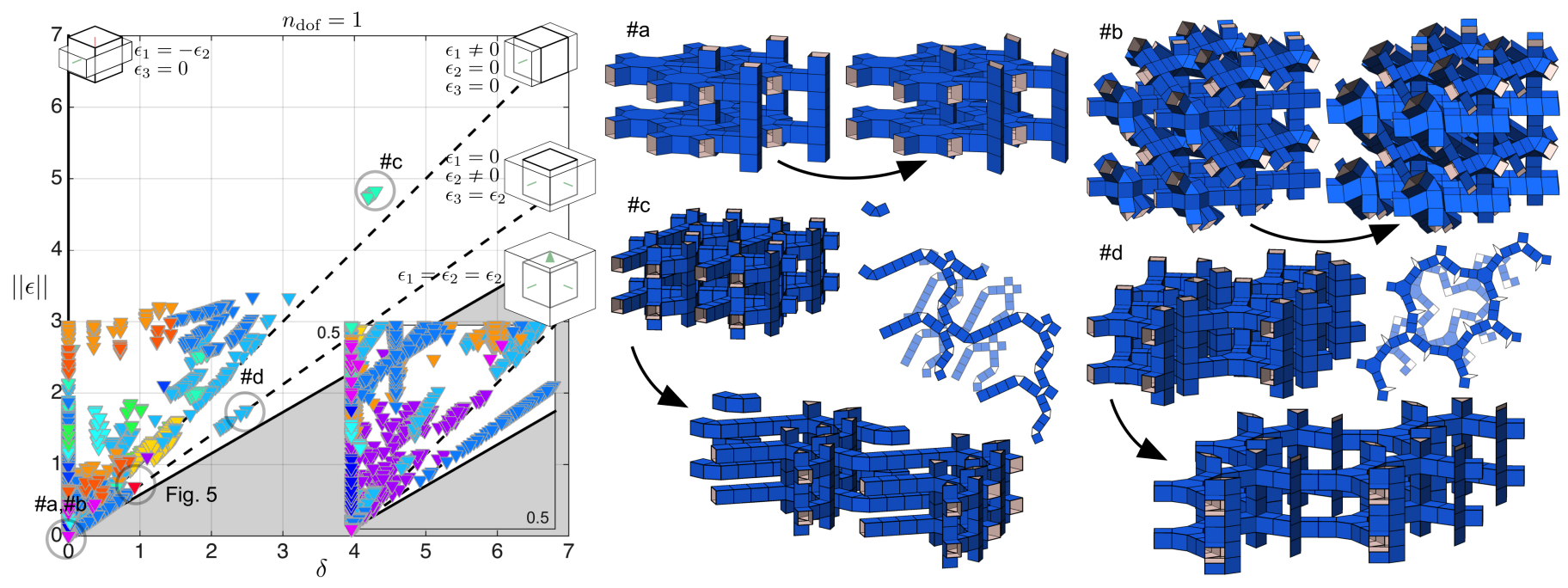

Figure 6: Deformation modes of 3D prismatic architected materials with enhanced reconfigurability. Relation between the volumetric strain, $\delta$, and the magnitude of the principal strains, $\|\epsilon\|$, for all the architected materials characterized by $n_{\mathrm{dof}}=1$. The color of the markers refers to the uniform tessellation that has been used as a template, as shown in Fig. 3. Structures \#a and \#b (based on \#24 and \#9 respectively) are characterized by $\delta=\|\epsilon\|=0$ and experience internal rearrangements that do not alter their macroscopic shape. Structure \#c (based on \#16) only deforms in one direction (i.e. $\delta=4.21,\|\epsilon\|=4.76$ ), while \#d (based on \#14) experiences uniform biaxial extension (or contraction) (i.e. $\delta=2.45,\|\epsilon\|=1.73$ ). 